01

\title{
Эффективный способ нахождения матричных элементов оператора дипольного момента и поправок к энергии многоатомной молекулы с помощью теории возмущений в рамках формализма полиномов квантовых чисел
}

\author{
(С) М.Ю. Юрьев, В.М. Вахромов, А.О. Волощенко, Л.Б. Клинк \\ Институт квантовой фризики, Иркутский национальный исследовательский технический университет, \\ 664074 Иркутск, Россия \\ e-mail: mixailyu2012@yandex.ru
}

Поступила в редакцию 03.03.2021 г.

В окончательной редакции 26.03.2021 г.

Принята к публикации 30.03.2021 г.

В рамках формализма полиномов квантовых чисел получены ангармонические поправки к энергии и матричные элементы дипольного момента до второго порядка для многоатомных молекул, приведен подробный алгоритм расчета. Полученные результаты для матричных элементов фундаментального перехода хорошо согласуются с литературными данными.

Ключевые слова: теория возмущений, дипольный момент, многоатомные молекулы, ангармонические колебания.

DOI: $10.21883 / \mathrm{OS} .2021 .07 .51073 .1958-21$

\section{Введение}

Инфракрасная (ИК) спектроскопия - мощный инструмент для характеристики молекул малых и средних размеров. С увеличением разрешающей способности ИК спектрометров возрастает сложность корректного распознавания экспериментальных спектров многоатомных систем. Как правило, ИК спектры характеризуют колебательно-вращательные переходы внутри молекулы. Прямая задача ИК спектроскопии непосредственно связана с нахождением колебательных и вращательных матричных элементов, поскольку интенсивность вынужденных переходов в дипольном приближении пропорциональна квадрату матричного элемента функции дипольного момента. В большинстве случаев подобные вычисления выполнялись в рамках гармонического приближения, в котором потенциальная энергия считается квадратичной, а дипольный момент линейным по нормальным координатам. С помощью масштабирования гармонических частот [1] или силовых констант (во внутренних координатах или полусимметричных координатах [2-5]) достигается лучшее количественное согласие с экспериментальными частотами. Простейший метод учета „механического“ ангармонизма (кубические члены и члены более высокого порядка в потенциале) обеспечивает колебательная теория возмущений второго порядка (VPT2) [6-8]. Вычисления в рамках данной теории становятся все более распространенными в квантово-химических приложениях.

Использование теории возмущений для расчета механических и электрооптических поправок к энергии и матричным элементам функции дипольного момента двухатомных молекул впервые представлено в работе [9], в которой были получены матричные элементы для переходов, связанных с основным колебательным состоянием молекулы. В последующих работах было показано, что отличные от нуля матричные элементы функции дипольного момента обладают зависимостью от квантового числа произвольного состояния, для которого рассматривается переход. Стоит отметить работу [10], где авторы изучили влияние ангармоничности на колебательные переходы двухатомных и многоатомных молекул, используя процедуру двойного контактного преобразования, основанную на канонической теории возмущений Ван Флека [11], до второго порядка. Уравнения, полученные методом двойного контактного преобразования [12-16], являются громоздкими и в работах [12-15] приводятся с опечатками или ошибками, за исключением статьи [16]. Сложность аналитического представления конечных выражений можно считать одной из причин сравнительно малого количества работ, посвященных расчетам интенсивности.

В данной работе сравнение проводится с результатами работ $[17,18]$. В цитированных работах авторы отказались от процедуры контактного преобразования, применив к проблеме теорию возмущений Релея-Шредингера. В работе [17] приводится уравнение для перехода $\left\langle 0\left|P_{i}\right| 0, \ldots, n_{i}=1, \ldots, 0\right\rangle$ для многоатомных молекул. Согласно [18], эта работа не лишена ошибок. При этом авторы подчеркивают, что их уравнение эквивалентно уравнению, приведенному в статье [16].

Несколько лет назад для решения задач с помощью теории возмущений был разработан формализм полино- 
мов квантовых чисел, в рамках которого $[19,20]$ были рассмотрены собственные колебания и электрооптика двухатомных молекул. Приведенные автором результаты вычислений для молекулы НІ и других галогеноводородов хорошо согласуются с экспериментальными данными. В работе [21] с помощью формализма проведены вычисления матричных элементов функции электрического дипольного момента и функции вращения в первом порядке теории возмущений для произвольной линейной многоатомной молекулы. К сожалению, приведенные в статье формулы содержат опечатки. В работах $[22,23]$ формализм полиномов квантовых чисел был успешно применен для определения уровней энергии атома водорода в однородном электрическом поле.

В данной работе в рамках формализма полиномов квантовых чисел получены формулы для расчета второй ангармонической поправки к энергии многоатомной молекулы и приведено выражение для горячих переходов $\left(n_{i}\left|P_{i}\right| n_{i}+1\right)$. Авторы посвящают выполненное исследование Михаилу Олеговичу Буланину в год его 90-летия.

\section{Теория}

Формализм полиномов квантовых чисел подробно описан в монографиях [24,25]. Представим гамильтониан системы как сумму невозмущенного гамильтониана и возмущения:

$$
H=H_{0}+\sum_{p>0} G_{p} \lambda^{p}
$$

где $H_{0}$ - гармонический гамильтониан, которому соответствует энергия $E_{n}^{0}$ и вектор состояния $|n\rangle, \lambda$ - малый параметр, характеризующий возмущение $G_{p}$, которое можно представить в виде ряда:

$$
G_{p}=\sum_{\left(j_{1}, j_{2}, \ldots, j_{r}\right) p+2} a_{j_{1}, j_{2}, \ldots, j_{r}} \xi_{1}^{j_{1}} \xi_{2}^{j_{2}} \ldots \xi_{r}^{j_{r}},
$$

где $a_{j_{1}, j_{2}, \ldots, j_{r}}$ - безразмерные ангармонические силовые постоянные, включающие в себя множитель $\frac{2^{-\frac{p+2}{2}}}{j_{1} ! j_{2} ! \ldots j_{r} !}$ (выражение $\left(j_{1}, j_{2}, \ldots, j_{r}\right) p+2$ под знаком суммы означает, что суммирование идет по индексам $j_{1}, j_{2}, \ldots, j_{r}$ при условии, что $\left.j_{1}+j_{2}+\cdots+j_{r}=p+2\right), \xi_{s}-$ более удобная вибрационная переменная, равная

$$
\xi_{s}=\eta_{s}+\eta_{s}^{+}=q_{s} \sqrt{2}
$$

где $\eta_{s}$ и $\eta_{s}^{+}$- операторы рождения и уничтожения для $s$-й компоненты соответственно, $q_{s}$ - нормальные координаты, $s=1,2,3, \ldots, r$.

Согласно теории возмущений, искомая энергия $E_{n}(\lambda)$ и точное состояние, которое мы обозначим как $\mid n, \lambda)$, могут быть записаны посредством введения разложений:

$$
E_{n}(\lambda)=E_{n}^{0}+\sum_{\alpha>0} \lambda^{\alpha} E_{n}^{\alpha}
$$

$$
\mid n, \lambda)=|n\rangle+\sum_{\alpha>0} \lambda^{\alpha}|n, \alpha\rangle,
$$

где $E_{n}^{\alpha}$ и $|n, \alpha\rangle-$ поправки к энергии гармонического осциллятора и к вектору состояния порядка $\alpha$ соответственно.

Поправки $E_{n}^{\alpha}$ и $|n, \alpha\rangle$ имеют вид

$$
\begin{gathered}
E_{n}^{\alpha}=\frac{1}{\alpha} \sum_{(p \beta \gamma) \alpha} p\left\langle n, \beta\left|G_{p}\right| n, \gamma\right\rangle, \\
|n, \alpha\rangle=\frac{1}{\alpha} \sum_{(p q \beta \gamma v) \alpha} \sum_{m \neq n} p \Delta_{q}(n, m)\left\langle m, \beta\left|G_{p}\right| n, \gamma\right\rangle|m, v\rangle,
\end{gathered}
$$

где

$$
\begin{gathered}
\Delta_{q}(n, m)=\frac{1}{q} \frac{\partial^{q}}{\partial \lambda^{q}}\left[\frac{1}{E_{n}(\lambda)-E_{m}(\lambda)}\right]_{\lambda=0}, \\
\Delta_{0}(n, m)=\frac{1}{E_{n}^{0}-E_{m}^{0}}, \\
E_{n}^{0}=\hbar \sum_{i} \omega_{i}\left(n_{i}+\frac{1}{2}\right) .
\end{gathered}
$$

Система выражений (2), (3) является рекуррентной. Соответсвенно для расчета поправок более высоких порядков необходимо иметь поправки более низких порядков, которые будут иметь полиномиальный вид. Введем определение матричного элемента через полином:

$$
\left\langle n, \beta\left|\xi_{1}^{j_{1}} \xi_{2}^{j_{2}} \ldots \xi_{r}^{j_{r}}\right| n+k, \gamma\right\rangle=\sqrt{g_{n, n+k}} \Pi_{\beta \gamma}^{j}(n, n+k),
$$

где $g_{n, n+k}=(n+1)(n+2) \ldots(n+k), g_{n, n}=1$.

Представим поправку к энергии $E_{n}^{\alpha}$ из выражения (2) через полиномы $\Pi_{\beta \gamma}^{j}(n, n)$, подставив в него (4):

$$
E_{n}^{\alpha}=\frac{1}{\alpha} \sum_{(p \beta \gamma) \alpha} p \sum_{(j) p+2} a_{j} \Pi_{\beta \gamma}^{j}(n, n) .
$$

При этом удобно представлять сумму полиномов одного порядка в виде их свертки:

$$
\Pi_{(\beta \gamma) \alpha}^{j}(k)=\sum_{(\beta \gamma) \alpha} \Pi_{\beta \gamma}^{j}(n, n+k) .
$$

Формализм позволяет определить ненулевые матричные элементы по существующему правилу отбоpa [24]. Согласно данному правилу, свертки полиномов $\Pi_{(\beta, \gamma) \alpha}^{j}(k)$ отличны от нуля лишь в том случае, если $\sum_{i} k_{i}$ имеет четность числа $\gamma+\beta+\sum_{i} j_{i}$. Например, рассмотрим первую поправку к энергии $E_{n}^{1}$, записав ее с помощью выражения (5),

$$
E_{n}^{1}=\sum_{(j) 3} a_{j} \Pi_{0,0}^{j}(n, n)=\left\langle n\left|G_{1}\right| n\right\rangle=0 .
$$

Данное выражение обращается в нуль, поскольку $\beta=0, \gamma=0$, а $\sum_{i} j_{i}=3$, соответственно число $\gamma+\beta+\sum_{i} j_{i}$ не имеет четность числа $\sum_{i} k_{i}=0$. 
Представим вторую поправку к энергии $E_{n}^{2}$ в виде свертки полиномов

$$
\begin{gathered}
E_{n}^{2}=\frac{1}{2} \sum_{(p \beta \gamma) 2} p \sum_{(j) p+2} a_{j} \Pi_{\beta \gamma}^{j}(n, n)=\frac{1}{2}\left(2 \sum_{(j) 4} a_{j} \Pi_{0,0}^{j}(n, n)\right. \\
\left.+\sum_{(j) 3} a_{j} \Pi_{(\beta \gamma) 1}^{j}(n, n)\right) .
\end{gathered}
$$

Согласно правилу отбора, ненулевой вклад будут вносить полиномы $\Pi_{0,0}^{4}(n, n), \quad \Pi_{0,0}^{2,2}(n, n), \Pi_{(\beta \gamma) 1}^{3}(n, n)$, $\Pi_{(\beta \gamma) 1}^{2,1}(n, n)$ и $\Pi_{(\beta \gamma) 1}^{1,1,1}(n, n)$. Далее произведем расчет второй поправки к энергии с последующей ее сверткой в полиномы.

\section{Расчет второй поправки для многоатомного случая}

Согласно (2), уравнение для второй поправки $(\alpha=2)$ к энергии многоатомной молекулы состоит из трех элементов, значения индексов в которых удовлетворяют условию $p+\beta+\gamma=2$. Распишем слагаемые в соответствии со значениями индексов:

$$
\begin{array}{cccc}
p=2, & \beta=0, \quad \gamma=0: & \left\langle n\left|G_{2}\right| n\right\rangle, \\
p=1, & \beta=1, \quad \gamma=0: & \left\langle n, 1\left|G_{1}\right| n\right\rangle, \\
p=1, & \beta=0, \quad \gamma=1: & \left\langle n\left|G_{1}\right| n, 1\right\rangle .
\end{array}
$$

Просуммируем эти выражения, получим:

$$
\begin{aligned}
E_{n}^{2}= & \frac{1}{2}\left\{\left\langle n, 1\left|G_{1}\right| n\right\rangle+\left\langle n\left|G_{1}\right| n, 1\right\rangle+2\left\langle n\left|G_{2}\right| n\right\rangle\right\} \\
& =\left\langle n, 1\left|G_{1}\right| n\right\rangle+\left\langle n\left|G_{2}\right| n\right\rangle,
\end{aligned}
$$

где слагаемые $\left\langle n, 1\left|G_{1}\right| n\right\rangle$ и $\left\langle n\left|G_{1}\right| n, 1\right\rangle$ тождественно равны в силу действительности волновых функций.

Используя выражения (1), получим следующие выражения для возмущений первого и второго порядков:

$$
\begin{aligned}
G_{1}= & \hbar \sum_{i} a_{i i i} \xi_{i}^{3}+\hbar \sum_{i j} a_{i i j} \xi_{i}^{2} \xi_{j}+\hbar \sum_{i j k} a_{i j k} \xi_{i} \xi_{j} \xi_{k} \\
G_{2}= & \hbar \sum_{i} a_{i i i i} \xi_{i}^{4}+\hbar \sum_{i j} a_{i i i j} \xi_{i}^{3} \xi_{j}+\hbar \sum_{i j} a_{i i j j} \xi_{i}^{2} \xi_{j}^{2} \\
& +\hbar \sum_{i j k} a_{i i j k} \xi_{i}^{2} \xi_{j} \xi_{k}+\hbar \sum_{i j k l} a_{i j k l} \xi_{i} \xi_{j} \xi_{k} \xi_{l} .
\end{aligned}
$$

Так же как и в случае с поправкой к энергии, согласно (3) первая поправка к вектору состояния будет иметь вид

$$
|n, 1\rangle=\sum_{m \neq n} \frac{\left\langle m\left|G_{1}\right| n\right\rangle|m\rangle}{E_{n}^{0}-E_{m}^{0}} .
$$

После выполнения всех действий с оператором $G_{1}$ имеем:

$$
|n, 1\rangle=\left|n_{x}, 1\right\rangle+|n, A\rangle+|n, B\rangle+|n, C\rangle,
$$

$$
\begin{gathered}
\left|n_{x}, 1\right\rangle=\sum_{x} \frac{a_{x x x}}{\omega_{x}}\left(\frac{\eta_{x}^{3}-\left(\eta_{x}^{+}\right)^{3}}{3}+3\left(n_{x} \eta_{x}-\left(n_{x}+1\right) \eta_{x}^{+}\right)\right)|n\rangle, \\
|n, A\rangle=\sum_{x y} \frac{2 a_{x x y}}{\omega_{y}}\left(n_{x}+\frac{1}{2}\right)\left(\eta_{y}-\eta_{y}^{+}\right)|n\rangle, \\
|n, B\rangle=\sum_{x y} a_{x x y}\left(\frac{\eta_{x}^{2} \eta_{y}-\left(\eta_{x}^{+}\right)^{2} \eta_{y}^{+}}{2 \omega_{x}+\omega_{y}}+\frac{\eta_{x}^{2} \eta_{y}^{+}-\left(\eta_{x}^{+}\right)^{2} \eta_{y}}{2 \omega_{x}-\omega_{y}}\right)|n\rangle, \\
|n, C\rangle=\sum_{x y z} a_{x y z}\left(\frac{\eta_{x} \eta_{y} \eta_{z}-\eta_{x}^{+} \eta_{y}^{+} \eta_{z}^{+}}{\omega_{x}+\omega_{y}+\omega_{z}}+\frac{\eta_{x} \eta_{y} \eta_{z}^{+}-\eta_{x}^{+} \eta_{y}^{+} \eta_{z}}{\omega_{x}+\omega_{y}-\omega_{z}}\right. \\
\left.+\frac{\eta_{x} \eta_{y}^{+} \eta_{z}-\eta_{x}^{+} \eta_{y} \eta_{z}^{+}}{\omega_{x}-\omega_{y}+\omega_{z}}+\frac{\eta_{x}^{+} \eta_{y} \eta_{z}-\eta_{x} \eta_{y}^{+} \eta_{z}^{+}}{-\omega_{x}+\omega_{y}+\omega_{z}}\right)|n\rangle .
\end{gathered}
$$

Подставив (8) в первое слагаемое выражения (7), найдем выражение для матричного элемента $\left\langle n, 1\left|G_{1}\right| n\right\rangle$ :

$$
\begin{aligned}
\left\langle n, 1\left|G_{1}\right| n\right\rangle= & \hbar \sum_{i}\left\langle n, 1\left|a_{i i i} \xi_{i}^{3}\right| n\right\rangle+\hbar \sum_{i j}\left\langle n, 1\left|a_{i i j} \xi_{i}^{2} \xi_{j}\right| n\right\rangle \\
& +\hbar \sum_{i j k}\left\langle n, 1\left|a_{i j k} \xi_{i} \xi_{j} \xi_{k}\right| n\right\rangle
\end{aligned}
$$

Необходимо учесть, что индексы $i, j, k$ и $x, y, z$ в уравнениях (8), (9) и (10) могут быть попарно или перекрестно равны. Матричные элементы, которые обращаются в нуль в силу ортонормированности векторов состояний $\left|n_{i}\right\rangle$ и $\left|n_{x}\right\rangle$ или $\left|n_{i}\right\rangle$ и $\left|n_{i} \pm k\right\rangle$, не приводятся. Далее для каждого конкретного ненулевого решения поясняется, при каких условиях оно было получено. Для краткости записи в обозначениях полагается $\bar{n}_{i}=n_{i}+\frac{1}{2}$.

Каждый матричный элемент выражения (11), в котором используется поправка к вектору состояния $|n, 1\rangle(10)$, в свою очередь раскладывается на более простые матричные элементы, представленные следующими выражениями:

$$
\begin{aligned}
& \sum_{i}\left\langle n, 1\left|a_{i i i} \xi_{i}^{3}\right| n\right\rangle=\sum_{i x}\left\langle n_{x}, 1\left|a_{i i i} \xi_{i}^{3}\right| n\right\rangle+\sum_{i}\left\langle n, A\left|a_{i i i} \xi_{i}^{3}\right| n\right\rangle \\
& +\sum_{i}\left\langle n, B\left|a_{i i i} \xi_{i}^{3}\right| n\right\rangle+\sum_{i}\left\langle n, C\left|a_{i i i} \xi_{i}^{3}\right| n\right\rangle \\
& \sum_{i j}\left\langle n, 1\left|a_{i i j} \xi_{i}^{2} \xi_{j}\right| n\right\rangle=\sum_{x i j}\left\langle n, 1\left|a_{i i j} \xi_{i}^{2} \xi_{j}\right| n\right\rangle \\
& +\sum_{i j}\left\langle n, A\left|a_{i i j} \xi_{i}^{2} \xi_{j}\right| n\right\rangle+\sum_{i j}\left\langle n, B\left|a_{i i j} \xi_{i}^{2} \xi_{j}\right| n\right\rangle \\
& +\sum_{i j}\left\langle n, C\left|a_{i i j} \xi_{i}^{2} \xi_{j}\right| n\right\rangle,
\end{aligned}
$$




$$
\begin{aligned}
& \sum_{i j k}\left\langle n, 1\left|a_{i j k} \xi_{i} \xi_{j} \xi_{k}\right| n\right\rangle=\sum_{x i j k}\left\langle n_{x}, 1\left|a_{i j k} \xi_{i} \xi_{j} \xi_{k}\right| n\right\rangle \\
& +\sum_{i j k}\left\langle n, A\left|a_{i j k} \xi_{i} \xi_{j} \xi_{k}\right| n\right\rangle+\sum_{i j k}\left\langle n, B\left|a_{i j k} \xi_{i} \xi_{j} \xi_{k}\right| n\right\rangle \\
& +\sum_{i j k}\left\langle n, C\left|a_{i j k} \xi_{i} \xi_{j} \xi_{k}\right| n\right\rangle .
\end{aligned}
$$

Ненулевой вклад будут вносить следующие слагаемые:

$$
\begin{gathered}
\sum_{i x}\left\langle n_{x}, 1\left|a_{i i i} \xi_{i}^{3}\right| n\right\rangle= \begin{cases}-\sum_{i} \frac{a_{i i i}^{2}}{\omega_{i}}\left(30 \bar{n}_{i}^{2}+\frac{7}{2}\right), & x=i \\
0, & x \neq i,\end{cases} \\
\sum_{i}\left\langle n, A\left|a_{i i i} \xi_{i}^{3}\right| n\right\rangle=-\sum_{i j} 12 \frac{a_{i i i} a_{j j i}}{\omega_{i}} \bar{n}_{i} \bar{n}_{j}, \quad y=i, \quad x=j, \\
\sum_{x i j}\left\langle n_{x}, 1\left|a_{i i j} \xi_{i}^{2} \xi_{j}\right| n\right\rangle=-\sum_{i j} 12 \frac{a_{i i j} a_{j j j}}{\omega_{j}} \bar{n}_{i} \bar{n}_{j}, \quad x=j, \\
\sum_{i j}\left\langle n, A\left|a_{i i j} \xi_{i}^{2} \xi_{j}\right| n\right\rangle= \begin{cases}-\sum_{i, j \neq x} a_{i i j} a_{x x j} \frac{4 \bar{n}_{i} \bar{n}_{x}}{\omega_{j}}, & x \neq i, y=j \\
-\sum_{i, j} a_{i i j}^{2} \frac{4 \bar{n}_{i}^{2}}{\omega_{j}}, & x=i, y=j,\end{cases} \\
\sum_{i j}\left\langle n, B\left|a_{i i j} \xi_{i}^{2} \xi_{j}\right| n\right\rangle=\sum_{i j} a_{i i j}^{2}\left(\frac{-\bar{n}_{i}^{2}-4 \bar{n}_{i} \bar{n}_{j}-\frac{3}{4}}{2 \omega_{i}+\omega_{j}}\right. \\
\left.+\frac{\bar{n}_{i}^{2}-4 \bar{n}_{i} \bar{n}_{j}+\frac{3}{4}}{2 \omega_{i}-\omega_{j}}\right), \quad x=i, \quad y=j, \\
\quad \sum_{i j k}\left\langle n, C\left|a_{i j k} \xi_{i} \xi_{j} \xi_{k}\right| n\right\rangle=\sum_{i j k} a_{i j k}^{2}\left(4 \bar{n}_{i} \bar{n}_{j}-\frac{1}{2}\right) \\
\quad \times \frac{\omega_{k}\left(\omega_{k}^{2}-\omega_{i}^{2}-\omega_{j}^{2}\right)}{\Delta_{i j k}}, x=i, \quad y=j, z=k,
\end{gathered}
$$

где $\Delta_{i j k}=\left(\omega_{i}+\omega_{j}+\omega_{k}\right)\left(\omega_{i}+\omega_{j}-\omega_{k}\right)\left(\omega_{i}-\omega_{j}+\omega_{k}\right) \times$ $\times\left(-\omega_{i}+\omega_{j}+\omega_{k}\right)$.

Используя (9), получим матричный элемент $\left\langle n\left|G_{2}\right| n\right\rangle$ :

$$
\begin{aligned}
\left\langle n\left|G_{2}\right| n\right\rangle= & \hbar \sum_{i}\left\langle n\left|a_{i i i i} \xi_{i}^{4}\right| n\right\rangle+\hbar \sum_{i j}\left\langle n\left|a_{i i i j} \xi_{i}^{3} \xi_{j}\right| n\right\rangle \\
& +\hbar \sum_{i j}\left\langle n\left|a_{i i j} \xi_{i}^{2} \xi_{j}^{2}\right| n\right\rangle+\hbar \sum_{i j k}\left\langle n\left|a_{i j k} \xi_{i}^{2} \xi_{j} \xi_{k}\right| n\right\rangle \\
& +\hbar \sum_{i j k l}\left\langle n\left|a_{i j k} \xi_{i} \xi_{j} \xi_{k} \xi_{l}\right| n\right\rangle .
\end{aligned}
$$

Выпишем ненулевые слагаемые:

$$
\sum_{i}\left\langle n\left|a_{i i i i} \xi_{i}^{4}\right| n\right\rangle=\sum_{i} 6 a_{i i i i}\left(\bar{n}_{i}^{2}+\frac{1}{4}\right),
$$

$$
\sum_{i j}\left\langle n\left|a_{i i j j} \xi_{i}^{2} \xi_{j}^{2}\right| n\right\rangle=\sum_{i j} 4 a_{i i j j} \bar{n}_{i} \bar{n}_{j}
$$

Сложив все полученные ранее ненулевые слагаемые, получим выражение для второй поправки к энергии (7):

$$
E_{n}^{2}=\hbar\left(\sum_{i j} a_{i i j}^{2}\left(\frac{-\bar{n}_{i}^{2}-4 \bar{n}_{i} \bar{n}_{j}-\frac{3}{4}}{2 \omega_{i}+\omega_{j}}+\frac{\bar{n}_{i}^{2}-4 \bar{n}_{i} \bar{n}_{j}+\frac{3}{4}}{2 \omega_{i}-\omega_{j}}\right)\right.
$$

$$
-\sum_{i} \frac{a_{i i i}^{2}}{\omega_{i}}\left(30 \bar{n}_{i}^{2}+\frac{7}{2}\right)-\sum_{i j k} a_{i i k} a_{j j k} \frac{4 \bar{n}_{i} \bar{n}_{j}}{\omega_{k}}-\sum_{i j} a_{i i j}^{2} \frac{4 \bar{n}_{i}^{2}}{\omega_{j}}
$$$$
+\sum_{i j k} a_{i j k}^{2}\left(4 \bar{n}_{i} \bar{n}_{j}-\frac{1}{2}\right) \frac{\omega_{k}\left(\omega_{k}^{2}-\omega_{i}^{2}-\omega_{j}^{2}\right)}{\Delta_{i j k}}
$$

$$
\left.+\sum_{i} 6 a_{i i i i}\left(\bar{n}_{i}^{2}+\frac{1}{4}\right)+\sum_{i j} 4 a_{i i j j} \bar{n}_{i} \bar{n}_{j}-\sum_{i j} 24 \frac{a_{i i i} a_{j j i}}{\omega_{i}} \bar{n}_{i} \bar{n}_{j}\right) .
$$

Слагаемые $\sum_{i}\left\langle n, A\left|a_{i i i} \xi_{i}^{3}\right| n\right\rangle$ и $\sum_{x i j}\left\langle n_{x}, 1\left|a_{i i j} \xi_{i}^{2} \xi_{j}\right| n\right\rangle$ в сумме дают вклад

$$
-\sum_{i j} 24 \frac{a_{i i i} a_{j j i}}{\omega_{i}} \bar{n}_{i} \bar{n}_{j}
$$

именно эти слагаемые не были учтены в работе [21]. Преобразуем и опустим численные константы, не зависящие от квантовых чисел:

$$
E_{n}^{2}=\hbar\left(\sum_{i} A_{i} \bar{n}_{i}^{2}+4 \sum_{i \neq j} A_{i j} \bar{n}_{i} \bar{n}_{j}\right)
$$

где

$$
\begin{aligned}
A_{i}= & 6\left(a_{i i i i}-5 \frac{a_{i i i}^{2}}{\omega_{i}}\right)+\sum_{j \neq i} a_{i i j}^{2} \frac{8 \omega_{i}^{2}-3 \omega_{j}^{2}}{\left(4 \omega_{i}^{2}-\omega_{j}^{2}\right) \omega_{j}}, \\
A_{i j}= & \left(a_{i i j j}-a_{i i j}^{2} \frac{4 \omega_{i}}{4 \omega_{i}^{2}-\omega_{j}^{2}}-6 \frac{a_{i i i} a_{j j i}}{\omega_{i}}\right) \\
& -\sum_{k \neq j \neq i}\left(\frac{a_{i i k} a_{j j k}}{\omega_{k}}+a_{i j k}^{2} \frac{\omega_{k}\left(\omega_{k}^{2}-\omega_{i}^{2}-\omega_{j}^{2}\right)}{\Delta_{i j k}}\right) .
\end{aligned}
$$

Полученные уравнения с точностью до обозначения ангармонических постоянных совпадают с уравнениями, приведенными в $[6,26]$. 
Представление второй поправки к энергии через полиномы квантовых чисел

Пользуясь формулой (6), распишем вторую поправку к энергии в полиномиальном виде:

$$
\begin{aligned}
E_{n}^{2}= & \hbar\left(\sum_{i}\left(\frac{1}{2} a_{i i i} \Pi_{(\alpha, \beta) 1}^{3}(n, n)+a_{i i i i} \Pi_{00}^{4}(n, n)\right)\right. \\
& +\sum_{i j}\left(\frac{1}{2} a_{i i j} \Pi_{(\alpha, \beta) 1}^{2,1}(n, n)+a_{i i j j} \Pi_{00}^{2,2}(n, n)\right) \\
& \left.+\frac{1}{2} \sum_{i j k} a_{i j k} \Pi_{(\alpha, \beta) 1}^{1,1,1}(n, n)\right)
\end{aligned}
$$

Приведем ненулевые свертки полиномов:

$$
\begin{aligned}
& \frac{1}{2} \Pi_{(\alpha, \beta) 1}^{3}(n, n)=\frac{\Pi_{(1,0)}^{3}(n, n)+\Pi_{(0,1)}^{3}(n, n)}{2} \\
& =\frac{\left\langle n, 1\left|\xi_{i}^{3}\right| n\right\rangle+\left\langle n\left|\xi_{i}^{3}\right| n, 1\right\rangle}{2} \\
& =-\frac{a_{i i i}}{\omega_{i}}\left(30 \bar{n}_{i}^{2}+\frac{7}{2}\right)-\sum_{j} 12 \frac{a_{j j i}}{\omega_{i}} \bar{n}_{i} \bar{n}_{j}, \\
& \frac{1}{2} \Pi_{(\alpha, \beta) 1}^{2,1}(n, n)=\frac{\Pi_{(1,0)}^{2,1}(n, n)+\Pi_{(0,1)}^{2,1}(n, n)}{2} \\
& =\frac{\left\langle n, 1\left|\xi_{i}^{2} \xi_{j}\right| n\right\rangle+\left\langle n\left|\xi_{i}^{2} \xi_{j}\right| n, 1\right\rangle}{2}=-12 \frac{a_{j j j}}{\omega_{j}} \bar{n}_{i} \bar{n}_{j} \\
& +a_{i i j}\left(\frac{\left(8 \omega_{i}^{2}-3 \omega_{j}^{2}\right) \bar{n}_{i}^{2}}{\left(4 \omega_{i}^{2}-\omega_{j}^{2}\right) \omega_{j}}-\frac{16 \omega_{i} \bar{n}_{i} \bar{n}_{j}}{4 \omega_{i}^{2}-\omega_{j}^{2}}\right)-\sum_{x} 4 a_{x x j} \frac{\bar{n}_{i} \bar{n}_{j}}{\omega_{j}} \\
& \frac{1}{2} \Pi_{(\alpha, \beta) 1}^{1,1,1}(n, n)=\frac{\Pi_{(1,0)}^{1,1,1}(n, n)+\Pi_{(0,1)}^{1,1,1}(n, n)}{2} \\
& =\frac{\left\langle n, 1\left|\xi_{i} \xi_{j} \xi_{k}\right| n\right\rangle+\left\langle n\left|\xi_{i} \xi_{j} \xi_{k}\right| n, 1\right\rangle}{2} \\
& =a_{i j k}\left(4 \bar{n}_{i} \bar{n}_{j}-\frac{1}{2}\right) \frac{\omega_{k}\left(\omega_{k}^{2}-\omega_{i}^{2}-\omega_{j}^{2}\right)}{\Delta_{i j k}}, \\
& \Pi_{00}^{4}(n, n)=\left\langle n\left|\xi_{i}^{4}\right| n\right\rangle=6 \bar{n}_{i}^{2}+\frac{3}{2}, \\
& \Pi_{00}^{2,2}(n, n)=\left\langle n\left|\xi_{i}^{2} \xi_{j}^{2}\right| n\right\rangle=4 \bar{n}_{i} \bar{n}_{j} .
\end{aligned}
$$

В выражении (16) опущен фактор $\sqrt{g(n, n)}=1$.

\section{Электрооптика}

Интенсивность вынужденных переходов из состояния $n$ в состояние $m$ в дипольном приближении определяется квадратом матричного элемента функции дипольного момента $d[14,15,17,21,23]$ :

$$
I_{n \rightarrow m} \sim \omega_{n, m} N\left(\omega_{n m}\right)|(n|d| m)|^{2} .
$$

Главной сложностью остается определение матричного элемента $(n|d| m)$, поскольку для правильного объяснения спектров наряду с механической ангармоничностью необходимо учитывать ангармоничность, обусловленную нелинейностью функции дипольного момента. Так, для произвольной многоатомной молекулы:

$$
d=\sum_{l} \sum_{\left(j_{1}, j_{2}, \ldots, j_{r}\right) l} \frac{2^{-l / 2}}{j_{1} ! j_{2} ! \ldots j_{r} !} d_{j_{1}, j_{2}, \ldots, j_{r}}^{(l)} \xi_{1}^{j_{1}} \xi_{2}^{j_{2}} \ldots \xi_{r}^{j_{r}}
$$

Рассмотрим выражение для горячих переходов $\left(n|d| n_{i}+1\right)$ многоатомной молекулы:

$$
\begin{aligned}
\left(n|d| n_{i}+1\right)= & \left\langle n|d| n_{i}+1\right\rangle+\left\langle n, 1|d| n_{i}+1\right\rangle \\
& +\left\langle n|d| n_{i}+1,1\right\rangle+\left\langle n, 1|d| n_{i}+1,1\right\rangle \\
& +\left\langle n, 2|d| n_{i}+1\right\rangle+\left\langle n|d| n_{i}+1,2\right\rangle
\end{aligned}
$$

Представим функцию дипольного момента $d$ в виде разложения в ряд Тейлора по колебательным переменным:

$$
\begin{aligned}
& d=d_{0}+\sum_{i} \frac{d_{i}^{\prime}}{\sqrt{2}} \xi_{i}+\sum_{i} \frac{d_{i i}^{\prime \prime}}{4} \xi_{i}^{2}+\sum_{i j} \frac{d_{i j}^{\prime \prime}}{2} \xi_{i} \xi_{j} \\
& +\sum_{i} \frac{d_{i i i}^{\prime \prime \prime}}{12 \sqrt{2}} \xi_{i}^{3}+\sum_{i j} \frac{d_{i i j}^{\prime \prime \prime}}{4 \sqrt{2}} \xi_{i}^{2} \xi_{i}+\sum_{i j k} \frac{d_{i j k}^{\prime \prime \prime}}{2 \sqrt{2}} \xi_{i} \xi_{j} \xi_{k} .
\end{aligned}
$$

Для краткости записи переопределим постоянные множители, используя обозначение $D_{j_{1}, j_{2}, \ldots j_{r}}$, которое содержит производные функции дипольного момента $d_{j_{1}, j_{2}, \ldots, j_{r}}^{(l)}$ и множитель $\frac{2^{-\frac{p}{2}}}{j_{1} ! j_{2} ! \ldots j_{r} !}:$

$$
\begin{aligned}
d= & d_{0}+\sum_{i} D_{i} \xi_{i}+\sum_{i} D_{i i} \xi_{i}^{2}+\sum_{i j} D_{i j} \xi_{i} \xi_{j}+\sum_{i} D_{i i i} \xi_{i}^{3} \\
& +\sum_{i j} D_{i i j} \xi_{i}^{2} \xi_{j}+\sum_{i j k} D_{i j k} \xi_{i} \xi_{j} \xi_{k}
\end{aligned}
$$

Отметим, что расчет слагаемого $\sum_{i j k}\left(n\left|D_{i j k} \xi_{i} \xi_{j} \xi_{k}\right| n_{i}+1\right)$ не приводится в последующих выкладках ввиду его громоздкости. Принимая во внимание только отличные от нуля матричные элементы, получаем следующее 
выражение во втором порядке теории возмущений:

$$
\begin{aligned}
& \left(n|d| n_{i}+1\right)=D_{i}\left\langle n\left|\xi_{i}\right| n_{i}+1\right\rangle+D_{i i i}\left\langle n\left|\xi_{i}^{3}\right| n+1\right\rangle \\
& +\sum_{j}\left[D _ { j } \left(\left\langle n, 1\left|\xi_{j}\right| n_{i}+1,1\right\rangle\right.\right. \\
& \left.+\left\langle n\left|\xi_{j}\right| n_{i}+1,2\right\rangle+\left\langle n, 2\left|\xi_{j}\right| n_{i}+1\right\rangle\right) \\
& +D_{j j}\left(\left\langle n, 1\left|\xi_{j}^{2}\right| n_{i}+1\right\rangle+\left\langle n\left|\xi_{j}^{2}\right| n_{i}+1,1\right\rangle\right) \\
& +D_{i j}\left(\left\langle n, 1\left|\xi_{i} \xi_{j}\right| n_{i}+1\right\rangle+\left\langle n\left|\xi_{i} \xi_{j}\right| n_{i}+1,1\right\rangle\right) \\
& \left.+D_{i j j}\left\langle n\left|\xi_{j}^{2} \xi_{i}\right| n+1\right\rangle\right] .
\end{aligned}
$$

Как видно из выражения (17), для дальнейших расчетов необходимо предварительно определить вторую поправку к вектору состояния $|n, 2\rangle$, которое можно получить, используя выражение (3):

$$
\begin{aligned}
& |n, 2\rangle=\frac{1}{2}\left(\Delta_{0}(n, m)\left\langle m, 1\left|G_{1}\right| n\right\rangle|m\rangle+\Delta_{0}(n, m)\left\langle m\left|G_{1}\right| n, 1\right\rangle|m\rangle\right. \\
& \left.+\Delta_{0}(n, m)\left\langle m\left|G_{1}\right| n\right\rangle|m, 1\rangle+2 \Delta_{0}(n, m)\left\langle m\left|G_{2}\right| n\right\rangle|m\rangle\right) .
\end{aligned}
$$

Конечный результат расчета вектора $|n, 2\rangle$ состоит из нескольких тысяч слагаемых, так как любой вектор вида $|n, a\rangle$ (3), (18) формируется с использованием векторов $|m\rangle$, которые в свою очередь принимают все возможные состояния $n_{1} \pm l_{1}, n_{2} \pm l_{2}, \ldots, n_{r} \pm l_{r}$, где $l_{1}+l_{2}+\cdots+l_{r} \leq 3 a$. Также необходимо учесть все возможные комбинации $i, j, k$ (из возмущения $G_{1}$ ) и $x, y, z$ (из вектора $|n, 1\rangle)$. О них мы упоминали ранее в тексте при разборе расчета второй поправки к энергии $E_{n}^{2}$.

Вычисления проводились с использованием библиотеки, реализованной на языке программирования Python. Результат гласит:

$\left(n|d| n_{i}+1\right)=A_{i}+\sum_{j \neq i}\left[B_{i j}+\sum_{k \neq j \neq i}\left(C_{i j k}+\sum_{l \neq k \neq j \neq i} E_{i j k l}\right)\right]$,

где

$$
\begin{aligned}
& A_{i}=\left(n_{i}+1\right)^{\frac{3}{2}}\left[\frac{22 D_{i} a_{i i i}{ }^{2}}{\omega_{i}^{2}}-\frac{6 D_{i} a_{i i i i}}{\omega_{i}}+3 D_{i i i}-\frac{20 D_{i i} a_{i i i}}{\omega_{i}}\right], \\
& B_{i j}=-\frac{40\left(n_{i}+1\right)^{\frac{3}{2}} D_{j} a_{i i j} a_{i i i} \omega_{j}}{\omega_{i}\left(\omega_{i}^{2}-\omega_{j}^{2}\right)} \\
& +\frac{12\left(n_{j}+\frac{1}{2}\right) D_{i} a_{j j i} a_{i i i} \sqrt{n_{i}+1}}{\omega_{i}{ }^{2}}+\frac{6 a_{i i i j} D_{j}\left(n_{i}+1\right)^{\frac{3}{2}} \omega_{j}}{\omega_{i}{ }^{2}-\omega_{j}{ }^{2}}
\end{aligned}
$$

$$
+\frac{64 a_{i i j}^{2} \sqrt{n_{i}+1} D_{i}}{\omega_{j}^{2} \omega_{i}\left(2 \omega_{i}-\omega_{j}\right)^{2}\left(2 \omega_{i}+\omega_{j}\right)^{2}}\left[\left(-n_{j}-\frac{1}{2}\right) \omega_{i}{ }^{5}\right.
$$$$
+\left(n_{i}+1\right) \omega_{i}^{4} \omega_{j}+\frac{5}{4} \omega_{j}^{2}\left(n_{j}+\frac{1}{2}\right) \omega_{i}^{3}
$$$$
\left.-\frac{3}{4}\left(n_{i}+1\right) \omega_{i}{ }^{2} \omega_{j}{ }^{3}-\frac{1}{8} \omega_{j}^{4}\left(n_{j}+\frac{1}{2}\right) \omega_{i}+\frac{3\left(n_{i}+1\right) \omega_{j}^{5}}{32}\right]
$$$$
+\frac{64 a_{i i j} a_{i j i} \omega_{j}^{3} D_{j} \sqrt{n_{i}+1}}{4 \omega_{i}^{5}-5 \omega_{i}^{3} \omega_{j}^{2}+\omega_{i} \omega_{j}^{4}}\left[\left(n_{i}+1\right) \omega_{i}^{3}+\frac{3}{2}\left(n_{j}+\frac{1}{2}\right)\right.
$$$$
\left.\times \omega_{j} \omega_{i}^{2}-\frac{3}{8}\left(n_{i}+1\right) \omega_{i} \omega_{j}^{2}-\frac{1}{4}\left(n_{j}+\frac{1}{2}\right)\right]
$$$$
+\frac{12\left(n_{j}+\frac{1}{2}\right) D_{i} a_{j i j} a_{i i j} \sqrt{n_{i}+1}}{\omega_{i} \omega_{j}}-\frac{\left(n_{j}+\frac{1}{2}\right) a_{i i j j} D_{i} \sqrt{n_{i}+1}}{\omega_{i}}
$$$$
+8 \sqrt{n_{i}+1}\left(n_{j}+\frac{1}{2}\right)\left(\frac{6 D_{j} a_{j j i i} \omega_{j}}{\omega_{i}^{2}-\omega_{j}^{2}}\right.
$$$$
\left.+\frac{D_{i} a_{j j i}^{2}\left(3 \omega_{i}^{2}-4 \omega_{j}^{2}\right) \omega_{j}}{\omega_{i}\left(\omega_{i}-2 \omega_{j}\right)^{2}\left(\omega_{i}+2 \omega_{j}\right)^{2}}+\frac{6\left(\omega_{i}^{2}-6 \omega_{j}^{2}\right) a_{j j j} D_{j} a_{j j i}}{\omega_{i}^{4}-5 \omega_{i}^{2} \omega_{j}^{2}+4 \omega_{j}{ }^{4}}\right)
$$$$
+2 \sqrt{n_{i}+1}\left[D_{i j j}\left(n_{j}+\frac{1}{2}\right)-\frac{6\left(n_{j}+\frac{1}{2}\right) D_{i j} a_{i j j}}{\omega_{j}}\right.
$$$$
+\frac{8\left(n_{j}+\frac{1}{2}\right) D_{j j} a_{i j i} \omega_{j}}{\omega_{i}^{2}-4 \omega_{j}^{2}}-\frac{4\left(n_{j}+\frac{1}{2}\right) D_{i i} a_{j j i}}{\omega_{i}}
$$$$
\left.-\frac{8 a_{i i j}\left(-\frac{3}{8}\left(n_{i}+1\right) \omega_{j}^{2}+\frac{1}{2} \omega_{i}\left(n_{j}+\frac{1}{2}\right) \omega_{j}+\left(n_{i}+1\right) \omega_{i}^{2}\right) D_{i j}}{4 \omega_{i}{ }^{2} \omega_{j}-\omega_{j}^{3}}\right],
$$

$$
\begin{gathered}
C_{i j k}=\frac{32 \sqrt{n_{i}+1}\left(\left(n_{i}+1\right) \omega_{i}^{2}+\frac{1}{2} \omega_{j}\left(n_{j}+\frac{1}{2}\right) \omega_{i}-\right.}{\omega_{j}\left(4 \omega_{i}^{2}-\omega_{j}^{2}\right)\left(\omega_{i}^{2}-\omega_{k}^{2}\right)} \\
-\frac{16\left(n_{k}-\frac{1}{2}\right) D_{j} a_{k k i} a_{i i j} \omega_{j} \sqrt{n_{i}+1}}{\omega_{i}^{3}-\omega_{i} \omega_{j}^{2}} \\
+\frac{4 a_{i i j}\left(n_{k}+\frac{1}{2}\right) a_{k k j} D_{i} \sqrt{n_{i}+1}}{\omega_{i} \omega_{j}} \\
-\frac{12 \sqrt{n_{i}+1} D_{k} a_{i j j} a_{i j k} \omega_{k}\left(2 n_{j}+1\right)}{\omega_{j}\left(\omega_{i}^{2}-\omega_{k}^{2}\right)} \\
-\frac{4 \sqrt{n_{i}+1} D_{k} a_{l l j} a_{i j k} \omega_{k}\left(2 n_{l}+1\right)}{\omega_{j}\left(\omega_{i}^{2}-\omega_{k}^{2}\right)}
\end{gathered}
$$




$$
\begin{aligned}
& -\frac{2 a_{i j k}^{2} D_{i} \sqrt{n_{i}+1}}{\begin{array}{c}
\omega_{i}\left(\omega_{i}+\omega_{j}-\omega_{k}\right)^{2}\left(\omega_{i}-\omega_{j}+\omega_{k}\right)^{2} \times \\
\times\left(\omega_{i}-\omega_{j}-\omega_{k}\right)^{2}\left(\omega_{i}+\omega_{j}+\omega_{k}\right)^{2}
\end{array}}\left[\left(-\frac{1}{2}-n_{k}\right) \omega_{j}^{7}\right. \\
& +\left(\frac{1}{2}+n_{j}\right) \omega_{k} \omega_{j}^{6}+5\left(\frac{1}{2}+n_{k}\right)\left(\omega_{i}^{2}+\frac{3}{5} \omega_{k}^{2}\right) \omega_{j}^{5} \\
& -7\left(\omega_{i}^{2}+\frac{3}{7} \omega_{k}^{2}\right)\left(\frac{1}{2}+n_{j}\right) \omega_{k} \omega_{j}^{4} \\
& -7\left(\omega_{i}^{4}-\frac{2}{7} \omega_{i}^{2}{\omega_{k}}^{2}+\frac{3}{7} \omega_{k}^{4}\right)\left(\frac{1}{2}+n_{k}\right) \omega_{j}^{3} \\
& +3\left(\omega_{i}^{4}+\omega_{k}^{4}+\frac{2}{3} \omega_{i}^{2} \omega_{k}^{2}\right)\left(\frac{1}{2}+n_{j}\right) \omega_{k} \omega_{j}^{2} \\
& +3\left(\omega_{i}+\omega_{k}\right)\left(\omega_{i}-\omega_{k}\right)\left(\frac{1}{2}+n_{k}\right)\left(\omega_{i}^{4}+2 \omega_{i}{ }^{2} \omega_{k}{ }^{2}\right. \\
& \left.-\frac{1}{3} \omega_{k}^{4}\right) \omega_{j}+3\left(\omega_{i}+\omega_{k}\right)^{2}\left(\omega_{i}-\omega_{k}\right)^{2}\left(\frac{1}{2}+n_{j}\right) \omega_{k} \\
& \left.\times\left({\omega_{i}}^{2}-\frac{1}{3} \omega_{k}^{2}\right)\right]-\frac{8 a_{j j k} a_{i j k} \omega_{j} D_{j} \sqrt{n_{i}+1}}{\begin{array}{c}
\left(\omega_{i}+\omega_{j}-\omega_{k}\right)\left(\omega_{i}-\omega_{j}+\omega_{k}\right)\left(\omega_{i}+\omega_{j}+\omega_{k}\right) \times \\
\times\left(\omega_{i}-\omega_{j}-\omega_{k}\right) \omega_{k}\left(\omega_{i}{ }^{2}-\omega_{j}{ }^{2}\right)
\end{array}} \\
& \times\left[3\left(n_{j}+\frac{1}{2}\right) \omega_{k}^{4}-2\left(n_{k}+\frac{1}{2}\right) \omega_{j} \omega_{k}^{3}-4\left(\frac{1}{2}+n_{j}\right)\right. \\
& \times\left(\omega_{i}^{2}+\omega_{j}^{2}\right) \omega_{k}^{2}-2\left(\omega_{i}-\omega_{j}\right)\left(\omega_{i}+\omega_{j}\right) \omega_{j} \\
& \left.\times\left(\frac{1}{2}+n_{k}\right) \omega_{k}+\left(\omega_{i}-\omega_{j}\right)^{2}\left(\omega_{i}+\omega_{j}\right)^{2}\left(\frac{1}{2}+n_{j}\right)\right] \\
& -\frac{16 a_{j j i} D_{j} \sqrt{n_{i}+1} a_{k k j}\left(\frac{1}{2}+n_{k}\right)}{\omega_{i}^{2}-\omega_{j}^{2}}-\frac{4 \sqrt{n_{i}+1}\left(n_{j}+\frac{1}{2}\right) D_{i k} a_{j j k}}{\omega_{k}} \\
& +\frac{32\left(\frac{1}{2}+n_{j}\right) a_{j j k} \omega_{k} D_{k} a_{j j i} \omega_{j} \sqrt{n_{i}+1}}{\left(\omega_{i}^{2}-4 \omega_{j}^{2}\right)\left(\omega_{i}^{2}-\omega_{k}^{2}\right)} \\
& +\frac{4 D_{k}\left(\frac{1}{2}+n_{j}\right) a_{j j i k} \omega_{k} \sqrt{n_{i}+1}}{\omega_{i}^{2}-\omega_{k}^{2}} \\
& +\frac{4 \sqrt{n_{i}+1} D_{j k} a_{i j k}}{\left(\omega_{i}-\omega_{j}+\omega_{k}\right)\left(\omega_{i}+\omega_{j}-\omega_{k}\right)\left(\omega_{i}-\omega_{j}-\omega_{k}\right) \times}\left[\left(-n_{k}-\frac{1}{2}\right) \omega_{j}{ }^{3}\right. \\
& +\omega_{k}\left(n_{j}+\frac{1}{2}\right) \omega_{j}^{2}+\left(\omega_{i}^{2}+\omega_{k}^{2}\right)\left(n_{k}+\frac{1}{2}\right) \omega_{j} \\
& \left.+\left(\omega_{i}+\omega_{k}\right)\left(n_{j}+\frac{1}{2}\right) \omega_{k}\left(\omega_{i}-\omega_{k}\right)\right]
\end{aligned}
$$

\section{Заключение}

Используя формализм полиномов квантовых чисел, было получено выражение для расчета второй поправки к энергии. Запись второй поправки к энергии $E_{n}^{2}(16)$ через полиномы квантовых чисел значительно упрощает дальнейший расчет. Формализм позволяет получить произвольную поправку посредством обращения к ранее рассчитанным полиномам, минуя промежуточные действия. Для численного расчета необходимо подставить в выражение значения частот и ангармонических силовых постоянных для рассматриваемой молекулы. Набор полиномов для одномерного случая приведен в [24].

В выражении (19) для определения матричных элементов горячих переходов вида $\left(n_{1}, n_{2}, \ldots, n_{i}, \ldots\right.$ $\left.\ldots|d| n_{1}, n_{2}, \ldots, n_{i}+1, \ldots\right)$ многоатомной молекулы слагаемое $A_{i}$ является решением одномерной задачи $\langle n|d| n+1\rangle$. Решение подобной задачи приведено в нескольких работах, например, в [19,20,23,24]. Сравнение выражения (19) было произведено с результатами работ $[17,18]$, в которых рассматривается переход вида $\left\langle n_{1}=0, n_{2}=0, \ldots n_{i}=0, \ldots|d| n_{1}=0, n_{2}=0, \ldots n_{i}=\right.$ $=1, \ldots\rangle$, что тождественно равно $\left\langle 0|d| n_{i}=1\right\rangle$ (в данных работах дипольный момент и его производные обозначаются через $P$ ). Предварительно в выражении (19) были вынесены численные множители из ангармонических постоянных $a_{i j k}$ и производных дипольного момента $D_{j_{1}, j_{2}, \ldots, j_{r}}$, возвращены исходные обозначения $d_{j_{1}, j_{2}, \ldots, j_{r}}^{(l)}$ и все $n_{k}$ положены равными нулю. Суммирование элементов по всем индексам кроме $i$ для каждого столбца таблицы (Приложение) даст результат перехода $\left\langle 0|d| n_{i}=1\right\rangle$. В результате такого преобразования выражение (19) становится полностью эквивалентно уравнению, полученному в работе [18], в работе [17] отсутствуют слагаемые $-\frac{\sqrt{2} k_{i i i i} P_{i}}{32 \omega_{i}}$ и $-\frac{\sqrt{2} k_{i i j} P_{i}}{32 \omega_{i}}$, возникающие из $D_{i}\left(\left\langle n\left|\xi_{i}\right| n_{i}+1,2\right\rangle+\left\langle n, 2\left|\xi_{i}\right| n_{i}+1\right\rangle\right)$ выражения (17).

\section{Благодарности}

Авторы выражают благодарность А.А. Вигасину за помощь в подготовке настоящей работы; Р.С. Букину („РТ-Техприемка“) за консультации в программной части; своему научному руководителю К.В. Казакову за ценные советы и поддержку в проведении исследования. 


\section{Сравнение результатов с литературными данными}

\begin{tabular}{|c|c|c|}
\hline Результаты данной работы & Stanton et al. [17] & Bloino and Barone $[18]$ \\
\hline$\frac{\sqrt{2} d_{i i i}}{8}$ & $\frac{\sqrt{2} P_{i i i}}{8}$ & $\frac{\sqrt{2} P_{i i i}}{8}$ \\
\hline$\frac{\sqrt{2} d_{i j}}{8}$ & $\frac{\sqrt{2} P_{i j i}}{8}$ & $\frac{\sqrt{2} P_{i j j}}{8}$ \\
\hline$\frac{\sqrt{2} a_{i j k} d_{j k}\left(\omega_{j}+\omega_{k}\right)}{8\left(\omega_{i}+\omega_{j}+\omega_{k}\right)\left(\omega_{i}-\omega_{j}-\omega_{k}\right)}$ & $\frac{\sqrt{2}\left(\omega_{j}+\omega_{k}\right) a_{i j k} P_{j k}}{8\left(\omega_{i}+\omega_{j}+\omega_{k}\right)\left(\omega_{i}-\omega_{j}-\omega_{k}\right)}$ & $\frac{\sqrt{2} k_{i j k} P_{j k}\left(\omega_{j}+\omega_{k}\right)}{8\left(\omega_{i}+\omega_{j}+\omega_{k}\right)\left(\omega_{i}-\omega_{j}-\omega_{k}\right)}$ \\
\hline$-\frac{5 \sqrt{2} d_{i i} a_{i i i}}{24 \omega_{i}}$ & $-\frac{5 \sqrt{2} a_{i i i} P_{i i}}{24 \omega_{i}}$ & $-\frac{5 \sqrt{2} k_{i i i} P_{i i}}{24 \omega_{i}}$ \\
\hline$-\frac{\sqrt{2} d_{i j} a_{i i j}\left(4 \omega_{i}+3 \omega_{j}\right)}{8 \omega_{j}\left(2 \omega_{i}+\omega_{j}\right)}$ & $-\frac{\sqrt{2}\left(3 \omega_{j}+4 \omega_{i}\right) a_{i j i} P_{j i}}{8\left(2 \omega_{i}+\omega_{j}\right) \omega_{j}}$ & $-\frac{\sqrt{2} k_{i i j} P_{i j}\left(4 \omega_{i}+3 \omega_{j}\right)}{8\left(2 \omega_{i}+\omega_{j}\right) \omega_{j}}$ \\
\hline$-\frac{\sqrt{2} d_{i i} a_{j i j}}{8 \omega_{i}}$ & $-\frac{\sqrt{2} a_{i j j_{i i}} P_{i i}}{8 \omega_{i}}$ & $-\frac{\sqrt{2} k_{i j j_{i i}} P_{i i}}{8 \omega_{i}}$ \\
\hline$\frac{\sqrt{2} d_{j j} a_{i j i} \omega_{j}}{4\left(\omega_{i}^{2}-4 \omega_{j}{ }^{2}\right)}$ & $\frac{\sqrt{2} \omega_{j} a_{i i j} P_{i j}}{4\left(\omega_{i}{ }^{2}-4 \omega_{j}{ }^{2}\right)}$ & $\frac{\sqrt{2} \omega_{j} a_{i i j} P_{i j}}{4\left(\omega_{i}{ }^{2}-4 \omega_{j}^{2}\right)}$ \\
\hline$-\frac{\sqrt{2} d_{i j} a_{i j j}}{8 \omega_{j}}$ & $-\frac{\sqrt{2} a_{i j j} P_{i j}}{8 \omega_{j}}$ & $-\frac{\sqrt{2} k_{i j i} P_{i j}}{8 \omega_{j}}$ \\
\hline$-\frac{\sqrt{2} d_{i k} a_{j j k}}{8 \omega_{k}}$ & $-\frac{\sqrt{2} a_{k i j} P_{i k}}{8 \omega_{k}}$ & $-\frac{\sqrt{2} k_{k i j} P_{i k}}{8 \omega_{k}}$ \\
\hline$-\frac{d_{i} a_{i i i i} \sqrt{2}}{32 \omega_{i}}$ & - & $-\frac{\sqrt{2} k_{i i i} P_{i}}{32 \omega_{i}}$ \\
\hline$-\frac{d_{i} a_{i i j j} \sqrt{2}}{32 \omega_{i}}$ & - & $-\frac{\sqrt{2} k_{i i j} P_{i}}{32 \omega_{i}}$ \\
\hline$\frac{\omega_{j} a_{i i i j} d_{j} \sqrt{2}}{8\left(\omega_{i}^{2}-\omega_{j}^{2}\right)}$ & $\frac{\sqrt{2} a_{i i i j} P_{j} \omega_{j}}{8\left(\omega_{i}{ }^{2}-\omega_{j}{ }^{2}\right)}$ & $\frac{\sqrt{2} \omega_{j} k_{i j i i} P_{j}}{8\left(\omega_{i}{ }^{2}-\omega_{j}{ }^{2}\right)}$ \\
\hline$\frac{d_{j} a_{i j i j i} \omega_{j} \sqrt{2}}{8\left(\omega_{i}^{2}-\omega_{j}^{2}\right)}$ & $\frac{\sqrt{2} a_{i j i j} P_{j} \omega_{j}}{8\left(\omega_{i}^{2}-\omega_{j}^{2}\right)}$ & $\frac{\sqrt{2} \omega_{j} k_{i j i j P} P_{j}}{8\left(\omega_{i}{ }^{2}-\omega_{j}{ }^{2}\right)}$ \\
\hline$\frac{a_{i j i k} \omega_{k} d_{k} \sqrt{2}}{8\left(\omega_{i}^{2}-\omega_{k}{ }^{2}\right)}$ & $\frac{\sqrt{2} a_{i j i k} P_{k} \omega_{k}}{8\left(\omega_{i}{ }^{2}-\omega_{k}{ }^{2}\right)}$ & $\frac{\sqrt{2} \omega_{k} k_{i k j i j} P_{k}}{8\left(\omega_{i}^{2}-\omega_{k}^{2}\right)}$ \\
\hline$\frac{11 d_{i} a_{i i i}^{2} \sqrt{2}}{288 \omega_{i}^{2}}$ & $\frac{11 \sqrt{2} a_{i i i}^{2} P_{i}}{288 \omega_{i}^{2}}$ & $\frac{11 \sqrt{2} k_{i i i}{ }^{2} P_{i}}{288 \omega_{i}{ }^{2}}$ \\
\hline$\frac{d_{i} a_{i j i} a_{i i i} \sqrt{2}}{32 \omega_{i}^{2}}$ & $\frac{\sqrt{2} a_{i i i} a_{i j i} P_{i}}{32 \omega_{i}{ }^{2}}$ & $\frac{\sqrt{2} k_{i i i} k_{i j i} P_{i}}{32 \omega_{i}^{2}}$ \\
\hline$\frac{5 \omega_{j} a_{i i i} a_{i i j} d_{j} \sqrt{2}}{24\left(\omega_{j}^{2}-\omega_{i}^{2}\right) \omega_{i}}$ & $\frac{5 \sqrt{2} a_{i i j} a_{i i i} P_{j} \omega_{j}}{24 \omega_{i}\left(\omega_{j}^{2}-\omega_{i}^{2}\right)}$ & $\frac{5 \sqrt{2} k_{i j i} k_{i i i} P_{j} \omega_{j}}{24\left(\omega_{j}^{2}-\omega_{j}^{2}\right) \omega_{i}}$ \\
\hline$d_{i}\left(4 \omega_{j} \omega_{i}^{2}+10 \omega_{j}{ }^{2} \omega_{i}+3 \omega_{j}{ }^{3}-4 \omega_{i}^{3}\right) a_{i i j}{ }^{2} \sqrt{2}$ & $P_{i} a_{i i j}{ }^{2}\left(4 \omega_{i}^{2} \omega_{j}+10 \omega_{i} \omega_{j}{ }^{2}+3 \omega_{j}{ }^{3}-4 \omega_{i}{ }^{3}\right) \sqrt{2}$ & $\sqrt{2} k_{i i j}{ }^{2}\left(4 \omega_{i}{ }^{2} \omega_{j}+10 \omega_{i} \omega_{j}{ }^{2}+3 \omega_{j}{ }^{3}-4 \omega_{i}{ }^{3}\right) P_{i}$ \\
\hline $32 \omega_{i}\left(\omega_{j}+2 \omega_{i}\right)^{2} \omega_{j}^{2}$ & $32 \omega_{i} \omega_{j}^{2}\left(2 \omega_{i}+\omega_{j}\right)^{2}$ & $32 \omega_{i} \omega_{j}^{2}\left(2 \omega_{i}+\omega_{j}\right)^{2}$ \\
\hline$\frac{a_{i i j} d_{j} a_{j i j}\left(\omega_{j}+4 \omega_{i}\right) \sqrt{2}}{8 \omega_{i}\left(\omega_{j}-\omega_{i}\right)\left(\omega_{j}+2 \omega_{i}\right)}$ & $\frac{\sqrt{2}\left(4 \omega_{i}+\omega_{j}\right) P_{j} a_{i j i} a_{j j i}}{8 \omega_{i}\left(2 \omega_{i}+\omega_{j}\right)\left(-\omega_{i}+\omega_{j}\right)}$ & $\frac{\sqrt{2} k_{i j i}\left(4 \omega_{i}+\omega_{j}\right) k_{j i i} P_{j}}{8 \omega_{i}\left(2 \omega_{i}+\omega_{j}\right)\left(-\omega_{i}+\omega_{j}\right)}$ \\
\hline$\frac{a_{i i j} a_{i j} d_{i} \sqrt{2}}{32 \omega_{j} \omega_{i}}$ & $\frac{\sqrt{2} a_{i i j} a_{i i j} P_{i}}{32 \omega_{i} \omega_{j}}$ & $\frac{\sqrt{2} k_{i j} k_{i j i j} P_{i}}{32 \omega_{i} \omega_{j}}$ \\
\hline$\frac{a_{i j i} d_{j} a_{i j j}\left(-\omega_{i}{ }^{2}+6 \omega_{j}{ }^{2}\right) \sqrt{2}}{8\left(\omega_{i}{ }^{4}-5 \omega_{j}{ }^{2} \omega_{i}{ }^{2}+4 \omega_{j}{ }^{4}\right)}$ & $\frac{\sqrt{2}\left(-\omega_{i}{ }^{2}+6 \omega_{j}^{2}\right) P_{j} a_{i j i} a_{i j i}}{8\left(\omega_{i}^{4}-5 \omega_{j}{ }^{2} \omega_{i}{ }^{2}+4 \omega_{j}{ }^{4}\right)}$ & $\frac{\sqrt{2} k_{i i j} k_{i j j} P_{j}\left(-\omega_{i}{ }^{2}+6 \omega_{j}{ }^{2}\right)}{8\left(\omega_{i}{ }^{4}-5 \omega_{j}{ }^{2} \omega_{i}{ }^{2}+4 \omega_{j}{ }^{4}\right)}$ \\
\hline$\frac{d_{i} a_{j j i}{ }^{2} \omega_{j}\left(4 \omega_{j}^{2}-3 \omega_{i}^{2}\right) \sqrt{2}}{16 \omega_{i}\left(\omega_{i}^{2}-4 \omega_{j}^{2}\right)^{2}}$ & $\frac{\sqrt{2} a_{i j j} a_{i j i} P_{i} \omega_{j}\left(4 \omega_{j}^{2}-3 \omega_{i}^{2}\right)}{16 \omega_{i}\left(\omega_{i}{ }^{2}-4 \omega_{j}{ }^{2}\right)^{2}}$ & $\frac{k_{i j i}^{2} \sqrt{2}\left(4 \omega_{j}^{2}-3 \omega_{i}^{2}\right) P_{i} \omega_{j}}{16 \omega_{i}\left(\omega_{i}{ }^{2}-4 \omega_{j}^{2}\right)^{2}}$ \\
\hline$\frac{a_{i j k}\left(3 \omega_{j}+4 \omega_{i}\right) d_{k} a_{i i j} \omega_{k} \sqrt{2}}{8\left(\omega_{k}^{2}-\omega_{i}^{2}\right)\left(\omega_{j}+2 \omega_{i}\right) \omega_{j}}$ & $\frac{\sqrt{2}\left(4 \omega_{i}+3 \omega_{j}\right) \omega_{k} a_{i j} a_{i j k} P_{k}}{8 \omega_{j}\left(2 \omega_{i}+\omega_{j}\right)\left(\omega_{k}^{2}-\omega_{i}^{2}\right)}$ & $\frac{\sqrt{2} k_{i j i}\left(4 \omega_{i}+3 \omega_{j}\right) k_{k j i} \omega_{k} P_{k}}{8 \omega_{j}\left(2 \omega_{i}+\omega_{j}\right)\left(\omega_{k}^{2}-\omega_{i}^{2}\right)}$ \\
\hline$\frac{d_{j} a_{i j j} a_{k k i} \omega_{j} \sqrt{2}}{8 \omega_{i}\left(\omega_{j}^{2}-\omega_{i}^{2}\right)}$ & $\frac{\sqrt{2} a_{i j i} a_{k k i} P_{j} \omega_{j}}{8 \omega_{i}\left(\omega_{j}^{2}-\omega_{i}^{2}\right)}$ & $\frac{\sqrt{2} k_{i j i} k_{k k i} P_{j} \omega_{j}}{8\left(\omega_{j}^{2}-\omega_{i}^{2}\right) \omega_{i}}$ \\
\hline$\frac{d_{j} a_{k k j} a_{j i j} \sqrt{2}}{8\left(\omega_{j}^{2}-\omega_{i}^{2}\right)}$ & $\frac{a_{i j j} a_{k k j} P_{j} \sqrt{2}}{8\left(\omega_{j}^{2}-\omega_{i}^{2}\right)}$ & $\frac{\sqrt{2} k_{i j} k_{k k j} P_{j}}{8\left(\omega_{j}{ }^{2}-\omega_{i}^{2}\right)}$ \\
\hline$\frac{a_{i i j} a_{k k j} d_{i} \sqrt{2}}{32 \omega_{j} \omega_{i}}$ & $\frac{\sqrt{2} a_{i i j} a_{k k j} P_{i}}{32 \omega_{i} \omega_{j}}$ & $\frac{\sqrt{2} k_{i i j} k_{k k j} P_{i}}{32 \omega_{i} \omega_{j}}$ \\
\hline$\frac{a_{i j i} d_{k} a_{i j k} \omega_{k} \sqrt{2}}{8 \omega_{j}\left(\omega_{k}^{2}-\omega_{i}^{2}\right)}$ & $\frac{\sqrt{2} a_{i j k} a_{i j 2} P_{k} \omega_{k}}{8 \omega_{j}\left(\omega_{k}{ }^{2}-\omega_{i}^{2}\right)}$ & $\frac{\sqrt{2} k_{i k j} k_{i j i} P_{k} \omega_{k}}{8 \omega_{j}\left(\omega_{k}^{2}-\omega_{i}^{2}\right)}$ \\
\hline$\frac{a_{j j k} \omega_{j} d_{k} a_{j i i} \omega_{k} \sqrt{2}}{4\left(4 \omega_{j}^{2}-\omega_{i}^{2}\right)\left(\omega_{k}^{2}-\omega_{i}^{2}\right)}$ & $\frac{\sqrt{2} a_{i j i} a_{i j k} P_{k} \omega_{j} \omega_{k}}{4\left(\omega_{k}^{2}-\omega_{i}^{2}\right)\left(4 \omega_{j}{ }^{2}-\omega_{i}{ }^{2}\right)}$ & $\frac{\sqrt{2} k_{i j i} k_{k j i} P_{k} \omega_{j} \omega_{k}}{4\left(4 \omega_{j}^{2}-\omega_{i}^{2}\right)\left(\omega_{k}^{2}-\omega_{i}^{2}\right)}$ \\
\hline$\frac{d_{i} a_{i j k}{ }^{2}\left(\omega_{j}+\omega_{k}\right)\left(-3 \omega_{i}{ }^{2}+\omega_{j}{ }^{2}+2 \omega_{k} \omega_{j}+\omega_{k}{ }^{2}\right) \sqrt{2}}{32\left(\omega_{i}-\omega_{j}-\omega_{k}\right)^{2}\left(\omega_{i}+\omega_{j}+\omega_{k}\right)^{2} \omega_{i}}$ & $\frac{\sqrt{2} a_{i j k}{ }^{2} P_{i}\left(\omega_{j}+\omega_{k}\right)\left(\omega_{j}{ }^{2}+2 \omega_{j} \omega_{k}+\omega_{k}{ }^{2}-3 \omega_{i}{ }^{2}\right)}{32 \omega_{i}\left(\omega_{i}+\omega_{j}+\omega_{k}\right)^{2}\left(\omega_{i}-\omega_{j}-\omega_{k}\right)^{2}}$ & $\frac{\sqrt{2} k_{i j k}{ }^{2}\left(-3 \omega_{i}{ }^{2}+\omega_{j}{ }^{2}+2 \omega_{j} \omega_{k}+\omega_{k}{ }^{2}\right)\left(\omega_{j}+\omega_{k}\right) P_{i}}{32 \omega_{j}\left(\omega_{j}+\omega_{j}+\omega_{k}\right)^{2}\left(\omega_{i}-\omega_{j}-\omega_{k}\right)^{2}}$ \\
\hline$\frac{32\left(\omega_{i}-\omega_{j}-\omega_{k}\right)\left(\omega_{i}+\omega_{j}+\omega_{k}\right) \omega_{i}}{a_{i j k} d_{j} \omega_{j} a_{i j k}\left(\omega_{i}{ }^{2}-\omega_{j}{ }^{2}-4 \omega_{k} \omega_{j}-3 \omega_{k}{ }^{2}\right) \sqrt{2}}$ & $\frac{\left.J 2 \omega_{i}{ }^{2} \omega_{i}+\omega_{j}+\omega_{k}\right)\left(\omega_{i}-\omega_{j}-\omega_{k}\right)}{\left(\omega_{i}^{2}-\omega_{j}^{2}-4 \omega_{j} \omega_{k}-3 \omega_{k}^{2}\right) a_{i j k} a_{j j k} P_{j} \omega_{j} \sqrt{2}}$ & $\frac{2 \delta \omega_{i}\left(\omega_{i}+\omega_{j} \omega_{k}\right)\left(\omega_{i}-\omega_{j}-\omega_{k}\right)}{\sqrt{2} k_{i j k} k_{j j k} P_{j} \omega_{j}\left(\omega_{i}{ }^{2}-\omega_{j}-4 \omega_{j} \omega_{k}-3 \omega_{k}{ }^{2}\right)}$ \\
\hline$\overline{8\left(\omega_{j}^{2}-\omega_{i}{ }^{2}\right)\left(\omega_{i}-\omega_{j}-\omega_{k}\right) \omega_{k}\left(\omega_{i}+\omega_{j}+\omega_{k}\right)}$ & $\overline{8\left(\omega_{i}-\omega_{j}-\omega_{k}\right)\left(\omega_{i}+\omega_{j}+\omega_{k}\right) \omega_{k}\left(\omega_{j}{ }^{2}-\omega_{i}{ }^{2}\right)}$ & $8 \omega_{k}\left(\omega_{j}{ }^{2}-\omega_{i}{ }^{2}\right)\left(\omega_{i}-\omega_{j}-\omega_{k}\right)\left(\omega_{i}+\omega_{j}+\omega_{k}\right)$ \\
\hline$\frac{\left(\omega_{j}+\omega_{k}\right) a_{i j k} a_{j k l} d_{l} \omega_{l} \sqrt{2}}{8\left(\omega_{i}^{2}-\omega_{l}^{2}\right)\left(\omega_{i}+\omega_{j}+\omega_{k}\right)\left(\omega_{i}-\omega_{j}-\omega_{k}\right)}$ & $\frac{\sqrt{2} P_{l} \omega_{l} a_{j k k} a_{j k l}\left(\omega_{j}+\omega_{k}\right)}{8\left(\omega_{i}+\omega_{j}+\omega_{k}\right)\left(\omega_{i}-\omega_{j}-\omega_{k}\right)\left(\omega_{i}^{2}-\omega_{l}^{2}\right)}$ & $\frac{\sqrt{2} k_{i j k} k_{j k l} P_{l} \omega_{l}\left(\omega_{j}+\omega_{k}\right)}{8\left(\omega_{i}^{2}-\omega_{l}^{2}\right)\left(\omega_{i}+\omega_{j}+\omega_{k}\right)\left(\omega_{i}-\omega_{j}-\omega_{k}\right)}$ \\
\hline$\frac{d_{k} a_{l j} a_{i j k} \omega_{k} \sqrt{2}}{8 \omega_{j}\left(\omega_{k}^{2}-\omega_{i}^{2}\right)}$ & $\frac{\sqrt{2} a_{i k} a_{j l l} P_{k} \omega_{k}}{8 \omega_{j}\left(\omega_{k}{ }^{2}-\omega_{i}{ }^{2}\right)}$ & $\frac{\sqrt{2} k_{i j k} k_{l j} P_{k} \omega_{k}}{8 \omega_{j}\left(\omega_{k}^{2}-\omega_{i}^{2}\right)}$ \\
\hline
\end{tabular}




\section{Конфликт интересов}

Авторы заявляют, что у них нет конфликта интересов.

\section{Список литературы}

[1] Scott A.P., Radom L. // J. Phys. Chem. 1996. V. 100. N 41. doi 10.1021/jp960976r

[2] Pulay P., Fogarasi G., Pang F., Boggs J.E. // J. Am. Chem. Soc. 1979. V. 101. N 10. doi 10.1021/ja00504a009

[3] Rauhut G., Pulay P. // J. phys. Chem. 1995. V. 99. N 10. doi 10.1021/j100010a019

[4] Baker J., Jarzecki A.A., Pulay P. // J. Phys. Chem. 1998. V. 102. N 8. doi 10.1021/jp980038m

[5] Baker J., Pulay P. // J. Comput. Chem. 1998. V. 19. N 10. doi 10.1002/(SICI)1096-987X(19980730)19:10<1187::AIDJCC7>3.0.CO;2-I

[6] Mills I.M.. // Molecular Spectroscopy: Modern Research. 1972. V. 1.

[7] Kolomiütsova T., Lyaptsev A., Shchepkin D. // Optics and Spectroscopy. 2012. V. 88. N 5. doi 10.1134/1.626856

[8] Kolomiütsova T., Shchepkin D. // Optics and Spectroscopy. 1989. V. 66. N 5. P. 603.

[9] Dunham J.L. // Phys. Rev. 1930. V. 35. N 11. doi 10.1103/PhysRev.35.1347

[10] Geerlings P., Berckmans D., Figeys H.P. // J. Mol. Struct. 1979. V. 57. doi 10.1016/0022-2860(79)80254-9

[11] Van Vleck J.H. // Phys. Rev. 1929. V. 33. N 4. doi 10.1103/PhysRev.33.467

[12] Secroun C., Barbe A., Jouve P.J. // J. Molec. Spectrosc. 1973. V. 45. N 1. doi 10.1016/0022-2852(73)90170-7

[13] Overend $J . / /$ Vibrational Intensities and Infrared and Raman Spectroscopy. Amsterdam: Elsevier, 1982, 466 p.

[14] Geerlings P., Berckmans D., Figers H.P. // J. Molec. Struct. 1979. V. 57. doi 10.1016/0022-2860(79)80254-9

[15] Berckmans D., Figers H.P., Geerlings P. // J. Molec. Struct: THEOCHEM. 1986. V. 148. N 1-2. doi 10.1016/0166-1280(86)85007-2

[16] Willetts A., Handy N.C., GreenJr W.H., Jayatilaka D. // J. Phys. Chem. 1990. V. 94. N 14. doi 10.1021/j100377a038

[17] Vázquez J., Stanton J.F. // Mol. Phys. 2006. V. 104. N 3. doi 10.1080/00268970500290367

[18] Bloino Julien, Barone Vincenzo. // J. Chem. Phys. 2012. V. 136. N 12. doi 10.1063/1.3695210

[19] Kazakov K.V. // Opt. Spectrosc. 2004. V. 97. N 5. doi 10.1134/1.1828622

[20] Kazakov K.V. // Opt. Spectrosc. 2008. V. 104. N 4. doi 10.1134/S0030400X08040012

[21] Pogrebnyak M.A. // Optics and Spectroscopy. 2013. V. 114. N 5. doi 10.1134/S0030400X13050135

[22] Kazakov K.V. // J. Math. Phys. 2019. V. 60. N 10. doi 10.1063/1.5086981

[23] Kazakov K.V. // Annals of Physics. 2020. V. 414. doi 10.1016/j.aop.2020.168096

[24] Kazakov K.V. Quantum Theory of Anharmonic Effects in Molecules. Amsterdam: Elsevier, 2012. 232 p.

[25] Kazakov K.V. Uncommon Paths in Quantum Physics. Amsterdam: Elsevier, 2014. 206 p.

[26] Papoušek D., Aliev M.R.. Molecular Vibrational/Rotational Spectra. Amsterdam: Elsevier, 1982. 323 p. 